\title{
Structural Analysis of Liquid Metal Catalysts in Porous Silica Utilizing Nano-CT and Analytical Transmission Electron Microscopy
}

Janis Wirth ${ }^{1 *}$, Silvan Englisch ${ }^{1}$, Christian Wiktor ${ }^{1}$, Nicola Taccardi ${ }^{2}$, Benjamin Apeleo Zubiri ${ }^{1}$, Peter Wasserscheid $^{2}$ and Erdmann Spiecker ${ }^{1 *}$

1. Institute of Micro- and Nanostructure Research (IMN) \& Center for Nanoanalysis and Electron Microscopy (CENEM), Friedrich-Alexander University, Erlangen, Germany.

2. Chair of Chemical Reaction Engineering, Friedrich-Alexander University, Erlangen, Germany.

* Corresponding authors: janis.wirth@fau.de, erdmann.spiecker@fau.de

X-ray nano-tomography (Nano-CT) allows non-destructive three-dimensional (3D) investigations of materials across multiple length scales. The ZEISS Xradia 810 Ultra instrument in Erlangen uses Fresnel zone plate optics to achieve 3D resolutions down to $50 \mathrm{~nm}$ and can be equipped with a Zernike phase ring enabling phase contrast in addition to standard absorption contrast. While the latter is utilized for imaging of materials containing regions of sufficiently different densities (i.e. containing elements with sufficiently different atomic numbers Z), the former can be employed to study weakly absorbing materials (low Z) and to discriminate materials exhibiting similar densities (similar Z). Due to its highresolution capabilities and flexible imaging modes the instrument is perfectly suited for scale-bridging correlative 3D studies of functional and structural materials in combination with Scanning Electron Microscopy (SEM) and Transmission Electron Microscopy (TEM) techniques [1,2].

We report on correlative 3D studies of $\mathrm{Pd}-(\mathrm{Pt})-\mathrm{Ga}$ liquid metal catalysts (LMCs) which show outstanding performance as catalyst for alkane dehydrogenation and, in particular, high resistance against coking [3]. LMCs exhibit a complex material structure featuring a molecularly defined, catalytically active liquid film/droplet layer adsorbed on macro-porous silica templates. High-resolution $3 \mathrm{D}$ characterization across different length scales is required to reveal the complex pore morphology and the position of the catalytically active sites. The combination of absorption and phase contrast Nano-CT enables an independent 3D imaging of the metal droplets and the macro-porous silica network (Figure 1b-d). Tomographic reconstructions enable non-destructive characterization of the $3 \mathrm{D}$ distribution of individual metal droplets inside the porous network structure (Fig. 1d). SEM imaging reveals Ga droplets on the surface of the porous silica support (Fig. 2 a,b) but does not allow to clarify to which extend $\mathrm{Ga}$ and $\mathrm{Pd}(\mathrm{Pt})$ is incorporated in the bulk of the porous network.

The chemical composition of individual metal droplets inside the porous network can be characterized by site-specific Focused Ion Beam (FIB) sample preparation combined with high-resolution analytical TEM techniques (Fig. 2). High-resolution Scanning TEM (STEM) imaging shows a single Ga droplet and the corresponding Energy-Dispersive X-ray Spectroscopy (EDXS) map reveals that the particle exhibits a bimetallic structure with $\mathrm{Pd}(\mathrm{Pt})$-rich and Ga-rich parts occurring in direct contact to each other. Preparing such sample structures also enables in situ TEM heating experiments to simulate the process parameters similar to typical process temperatures of around $500{ }^{\circ} \mathrm{C}[3,4]$. 


\section{References:}

[1] Salter et al., Scientific Reports 7 (2017), p. 7332.

[2] Wirth et al., Microsc. Microanal. 24 (S2) (2018), p. 556.

[3] N Taccardi et al., Nat. Chem. 9 (2017), p. 862.

[4] The authors thank the DFG for financial support within the frameworks of the research training group GRK1896 "In situ Microscopy with Electrons, X-rays and Scanning Probes" and the project SP648/8 "High-resolution X-ray microscopy for correlative tomography, high throughput screening and in situ mechanical testing of structural and functional materials"
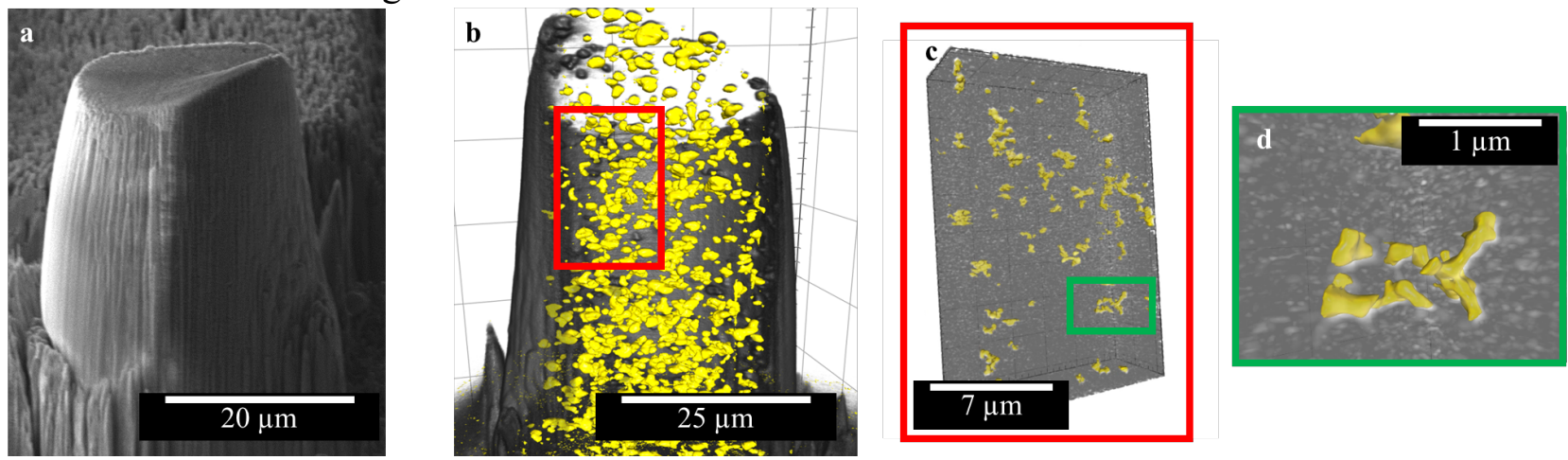

Figure 1. (a) SEM image of pillar structure prepared by FIB milling with a diameter of around $30 \mu \mathrm{m}$ suitable for Nano-CT. (b) 3D volume rendering of tomogram derived from large-field of view (LFOV) absorption contrast measurements reveals the position and approximate size of the Ga droplets (yellow) inside the porous silica support. (c, d) 3D volume renderings of tomogram obtained in high-resolution (HRES) mode (region marked in b) using phase contrast imaging unravels exact morphology of Ga droplets inside the porous glass network.
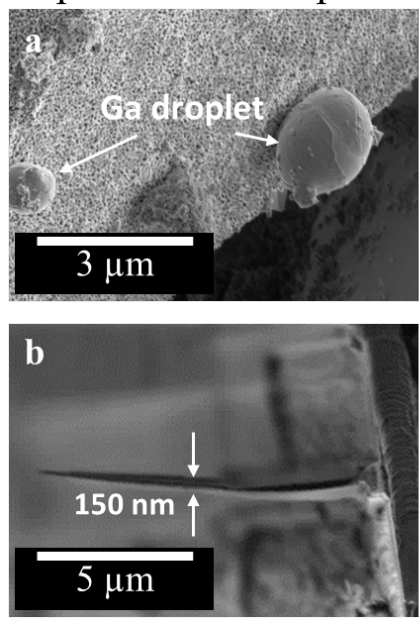
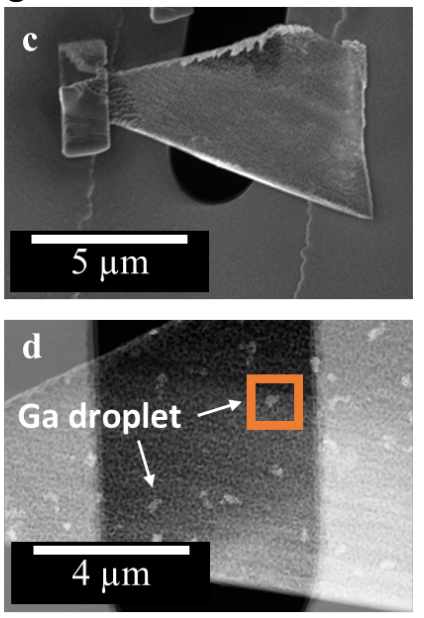
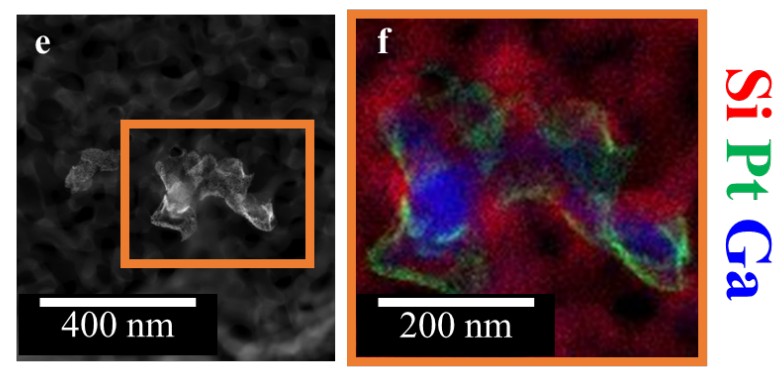

Figure 2. (a) SEM image of Ga droplets located on surface of porous silica. (b) TEM lamella prepared via FIB milling with a thickness of around $150 \mathrm{~nm}$. (c) SEM image of TEM lamella transferred to DENSsolution heating-chip, suitable for in situ TEM experiments. (d) High-angle annular dark-field STEM image of Ga droplets located in porous glass network. (e) STEM image of an exemplary single Ga droplet (marked in d) with bright contrast in outer regions. (f) STEM-EDXS map reveals chemical composition of the Pt-Ga droplet displayed in e). 